\title{
Contextual modulation of simultaneous associations
}

\author{
LOUIS D. MATZEL, JUAN CASTILLO, and RALPH R. MILLER \\ State University of New York at Binghamton, Binghamton, New York
}

\begin{abstract}
In four experiments with water-deprived rats, we examined the possibility that simultaneous associations between a conditioned stimulus (CS) and an unconditioned stimulus (US) were modulated or masked by context-US associations. In Experiment 1 we determined that simultaneous CS-US pairings administered in multiple contexts enhanced responding to the CS relative to training in a single context. A forward CS-US arrangement produced no such enhancement. In subsequent experiments, animals were trained in a single context. In Experiment 2, the number of CS-US pairings was varied and no inverse relationship between the response directly elicited by the training context and the response elicited by the CS was found. In Experiments 3 and 4 , we detected no effect on the response to the CS of extensive exposure to the training context before or after simultaneous CS-US pairings, although such latent inhibition or extinction treatment reduced the response elicited by the context. In total, these results indicate that the response to a simultaneously trained CS may be modulated by its training context, but that the response to the CS does not simply vary inversely with the response potential of that context.
\end{abstract}

When a conditioned stimulus (CS) and an unconditioned stimulus (US) are paired simultaneously, the CS typically elicits a weaker conditioned response than is elicited by the same CS when trained with a forward relationship to the US (e.g., Burkhardt \& Ayres, 1978; Pavlov, 1927). Although this simultaneous conditioning deficit typically is thought to represent a learning failure due to the poor information value of the CS (e.g., Cantor, 1981), studies of CS information value in classical conditioning that compare simultaneous and forward conditioning procedures are possibly confounded by differential conditioning of the training context. When a CS and US are presented simultaneously, no stimulus provides predictive information about the US; that is, the US is unsignaled. Consequently, the context might be expected to acquire a higher degree of associative strength than it has when the US is preceded (signaled) by a punctate cue (Randich \& LoLordo, 1979; Rescorla, 1984). An excitatory context is thought to impede both acquisition of CS-US associations (e.g., Randich \& LoLordo, 1979) and their expression (e.g., Matzel, Brown, \& Miller, 1987). In the present experiments we examined the possibility that the associative strength of the training context might modulate the response to a CS paired simultaneously with a US.

\section{EXPERIMENT 1}

\section{Method}

Subjects. Twenty-four male and 24 female adult Sprague-Dawley descended naive rats served as subjects. Body weights were 240-395 g for males and 195-280 $\mathrm{g}$ for females. The animals were singly housed in a vivarium maintained on a 16:8-h light:dark cycle, with experimental manipulations occurring near the middle of the light phase. The

This work was supported by NSF Grant BNS 86-00755. Address correspondence to R. R. Miller, Department of Psychology, SUNYBinghamton, Binghamton, NY 13901. animals had free access to food, whereas water availability outside the experimental sessions was limited to $10 \mathrm{~min} /$ day.

Apparatus. Four types of contexts (six of each) were used. Each chamber was individually housed in an environmental chest. Context A was a clear Plexiglas box $(22.75 \times 8.25 \times 13 \mathrm{~cm})$ with a floor of $0.48-\mathrm{cm}$ diameter steel rods. On a narrow wall, there was a water-filled lick tube connected to a lickometer. Context $\mathrm{B}$ in vertical profile was a truncated $\mathrm{V}$ (30 cm long, $28 \mathrm{~cm}$ high, and $21 \mathrm{~cm}$ wide at the top, $5.25 \mathrm{~cm}$ wide at the bottom), with black Plexiglas end walls. The floor and side walls were constructed of sheet metal with a $1.25-\mathrm{cm}$ gap centered lengthwise in the floor. A lick tube, attached to a lickometer, protruded through an end wall. Context $\mathrm{C}$ was a long, narrow rectangular enclosure $(50 \times 8 \times 59 \mathrm{~cm})$ with gray Plexiglas walls and an electrifiable $0.64-\mathrm{cm}$ grid floor. Context D was a large box $(24.1 \times 12.7 \times 20 \mathrm{~cm})$ with front and rear walls of clear Plexiglas, side walls of white Plexiglas, and a floor of $0.64-\mathrm{cm}$ steel rods. Equivalent animals had previously been found to discriminate between these contexts (Matzel et al., 1987). A 1500$\mathrm{Hz}$ tone, $12 \mathrm{~dB}(\mathrm{C})$ above background, could be delivered to all contexts.

Procedure. On Days 1 and 2, all subjects were acclimated to Contexts A and B for $20 \mathrm{~min}$, and latencies to complete Licks $0-25$ and 25-50 were recorded. Counterbalancing for weight, sex, and lick rates, the animals were then randomly assigned to four groups $(n s=12)$. Conditioning was conducted on Days 3-5. On these days, all animals spent 20 min in Contexts B, C, and D. Groups F-MC (forward-multiple contexts) and S-MC (simultaneous-multiple contexts) were placed in the contexts in the order $\mathrm{B}, \mathrm{C}$, and $\mathrm{D}$, where they received two randomly distributed 3-sec, 0.5-mA, constant-current footshocks in a different context on each day. For Group F-MC the shock was immediately preceded by a 3-sec tone that terminated at shock onset. For Group S-MC the tone and shock were simultaneous. Groups F-SC (forward-single context) and S-SC (simultaneous-single context) received the same forward or simultaneous tone-shock pairings, but always in Context $\mathrm{B}$. The order in which Groups F-SC and S-SC were placed in the three contexts was varied on each conditioning day to equate the time of conditioning with that of Groups F-MC and S-MC.

To ensure stable lick rates, all animals were placed in Context $\mathrm{A}$ for 20 min on Day 6 without any CS or US presentations. Latencies for Licks 0-25 and 25-50 were recorded. On Day 7 (CS test), all animals were placed in Context A and allowed to complete 25 licks, at which time the CS was presented and remained on until 25 additional licks had been completed. On Day 8 (Context B test), all animals were placed in Context B and latencies to complete 25 licks in the absence of any punctate stimulus were recorded. 


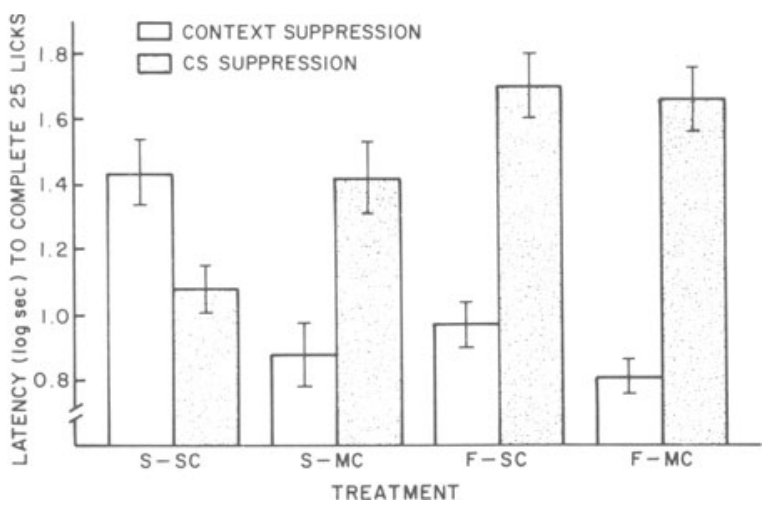

Figure 1. Mean response to the $\mathrm{CS}$ and to the training context in Experiment 1 after simultaneous (S) or forward (F) conditioning in a single context (SC) or in multiple contexts (MC). Bars indicate standard errors.

\section{Results and Discussion}

Latencies for Licks 0-25 and 25-50 were transformed to $\log$ latencies to permit the use of parametric statistics. There were no between-group differences for the first 25 licks in Contexts A and B prior to training or in Context $\mathrm{A}$ on the CS test day $(F \mathrm{~S}<1.20)$. An analysis of variance (ANOVA) of suppression to the CS indicated an effect of type of conditioning [simultaneous or forward; $F(1,44)=17.58, p<.001]$, but no effect of the number of training contexts ( 1 or $3 ; F=2.29$ ) (see Figure 1$)$. The conditioning $\times$ number interaction approached significance $[F(1,44)=3.11, p<.10]$. Planned comparisons indicated that Groups S-MC and S-SC differed from one another $[F(1,44)=5.26, p<.05]$. Groups F-MC and F-SC did not differ $(F<1.0)$.

The associative strength of Context $B$ was indicated by the latency to complete 25 licks on Day 8 (see Figure 1). An ANOVA indicated an effect of type of conditioning $[F(1,44)=8.39, p<.01]$, an effect of number of conditioning contexts $[F(1,44)=15.36, p<.001]$, and an interaction of these factors $[F(1,44)=4.73, p<.05]$.

These results suggest that simultaneous conditioning is enhanced by training in multiple contexts. This was specific to simultaneous conditioning, inasmuch as no such enhancement was evident with forward conditioning. Moreover, simultaneous conditioning produced stronger responding to the conditioning context than did forward conditioning. Since suppression to Context B was attenuated in animals trained with a simultaneous CS in multiple contexts, as opposed to a single context, and since these animals also exhibited an enhanced response to the $\mathrm{CS}$, it is possible that context-US associations are responsible for part of the simultaneous conditioning deficit.

\section{EXPERIMENT 2}

In Experiment 2 we directly examined the relationship between context-US associations and the response to a CS trained with a simultaneous US. Heth (1976) reported that simultaneous CSs elicit more responding early in training than after extensive training. Thus, as the response potential of the conditioning context increased in Experiment 1, it may have interfered with responding to the discrete CS. In Experiment 2 we tested this possibility by observing suppression to the CS (in a neutral context) and suppression to the training context after 2 , 8 , and 16 simultaneous CS-US pairings.

\section{Method}

Subjects and Apparatus. The subjects were 24 rats of the same description as those used in Experiment 1. The apparatus was that used as Contexts A and B of Experiment 1.

Procedure. All animals received lick shaping in Contexts A and B on Days 1 and 2 as in Experiment 1. On Days 3 and 4, conditioning was conducted in Context B during 60 -min sessions. Groups $1 / \mathrm{S}, 4 / \mathrm{S}$, and $8 / S(n s=8)$ received 1,4 , and 8 daily simultaneous CS-US pairings, respectively, using the parameters of Experiment 1. On Day 5, all animals received a 20-min session in Context A to ensure stable licking there. On Day 6, each subject was tested in Context A for lick suppression in response to the tone $\mathrm{CS}$ using the same procedure as in Experiment 1. On Day 7, lick suppression in response to Context B was indexed.

\section{Results}

No differences in lick rates were observed in Context A and Context B on Day 2, or prior to CS onset in Context A on Day $6(F \mathrm{~s}<1.0)$. Similarly, no differences were seen in response to the CS on Day $6(F=0.72)$. However, responses to Context $\mathrm{B}$ differed as a function of the number of CS-US pairings $[F(2,21)=3.34$, $p=.05$ ] (see Figure 2).

These results provide no evidence that the strength of the response to a simultaneous CS is inversely related to that elicited by its training context. Moreover, no inverse relationship was found between the number of CS-US pairings and the response to the CS. A loss of the small response to the CS that was observed might possibly have been obtained with more extensive training (Heth, 1976). Nevertheless, context-US associations were apparently

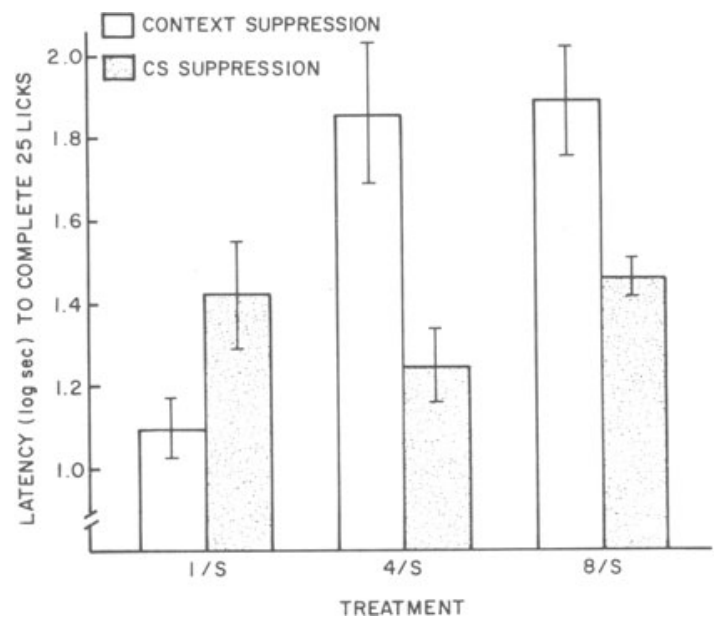

Figure 2. Mean response to the $\mathrm{CS}$ and to the training context in Experiment 2 after two simultaneous conditioning sessions consisting of one CS-US pairing per session (1/S), four pairings per session (4/S), or eight pairings per session (8/S). Bars indicate standard errors. 


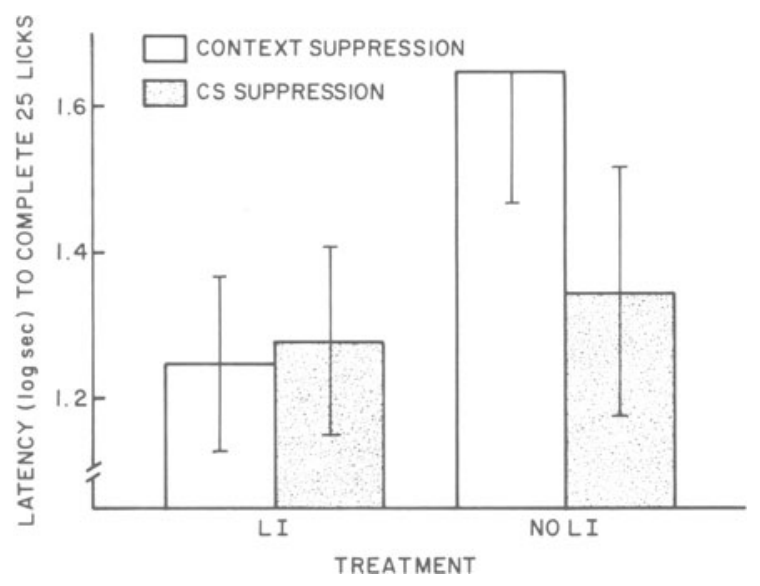

Figure 3. Mean response to the $\mathrm{CS}$ and to the training context in Experiment 3 after simultaneous conditioning preceded by latent inhibition (LI) or no latent inhibition (noLI) of the training context. Bars indicate standard errors.

asymptotic with both 8 and 16 CS-US pairings over the two conditioning days. Thus it is unlikely that any decrement in responding to the CS that might have occurred with additional pairings would be a simple consequence of increased context-US associations.

\section{EXPERIMENT 3}

Reduced acquisition of response strength occurs when a CS is extensively presented prior to CS-US pairings (Lubow, 1973). In Experiment 3 we used this phenomenon with respect to the training context to see whether the response strength of the context modulates responding to a punctate CS. Presumably, preexposure to the training context would slow acquisition of the context-US association, possibly allowing a CS presented simultaneously with a US to condition more fully.

\section{Method}

Subjects and Apparatus. The subjects were 12 rats of the same description as those used in Experiment 1, and the apparatus was that used as Contexts A and B of Experiment 1.

Procedure. All animals were acclimated and allowed to lick in Contexts A and B for 20 min on Days 1 and 2. On Days 3-12, Group LI (latent inhibition; $n=6$ ) was placed in Context $B$ for 60 min each day without presentation of discrete stimuli. Group noLI $(n=6)$ remained in the home cage. On Day 13, all animals were placed in Context B and received four simultaneous CS-US pairings, as described in Experiment 1 , distributed in a 60 -min session. All animals were reacclimated to Context A on Day 14 and were tested for suppression to the CS in Context A on Day 15. Latencies to complete 25 licks in Context B were recorded on Day 16.

\section{Results}

No differences existed in the latencies to complete 25 licks in Contexts A and B on Day 2, or in Context A prior to CS onset on the CS test day. Similarly, the two groups did not differ in response to the tone CS on Day 15 $(F=0.11)$ (see Figure 3). Group noLI exhibited a ten- dency toward greater suppression to Context $\mathrm{B}[F(1,10)$ $=3.67, p<.10]$.

Although extensive preexposure to the training context appears to have attenuated the degree to which that context accrued associative strength during simultaneous CS-US presentations, the attenuation of context-US associations had no effect on the degree to which the simultaneous CS controlled responding.

\section{EXPERIMENT 4}

The facilitated response observed in Experiment 1 to the simultaneous CS could be attributed to either learning or performance factors. In Experiment 4, we examined a factor that might be expected to influence only performance to a CS. To the extent that the training context was even weakly excitatory at the time of testing, it may have masked responding to the CS even though the CS was tested outside of the training context (Miller \& Schachtman, 1985). To examine this possibility, in Experiment 4 we extinguished the conditioning context after simultaneous CS-US pairings. Matzel et al. (1987) showed that this manipulation reduced a response deficit known to result from an excitatory training context, the US-preexposure deficit.

\section{Method}

Subjects and Apparatus. The subjects were 12 rats of the same description as those used in Experiment 1, and the apparatus was that used as Contexts $\mathrm{A}$ and $\mathrm{B}$ in Experiment 1.

Procedure. All animals were adapted to Contexts A and B on Days 1 and 2 as in Experiment 1. On Day 3, all animals were placed in Context $B$ and received four simultaneous CS-US pairings in a 60 -min session, using the parameters of Experiment 1. On Days 4-11, half of the animals (Group E [extinction], $n=6$ ) were placed in Context $B$ for 60 min each day without any CS or US presentations. This treatment was intended to extinguish any Context B-US association. The remaining animals (Group noE, $n=6$ ) remained in their home cages. On Day 12, all animals were placed in Context $\mathrm{A}$ for $20 \mathrm{~min}$ to ensure stable lick-

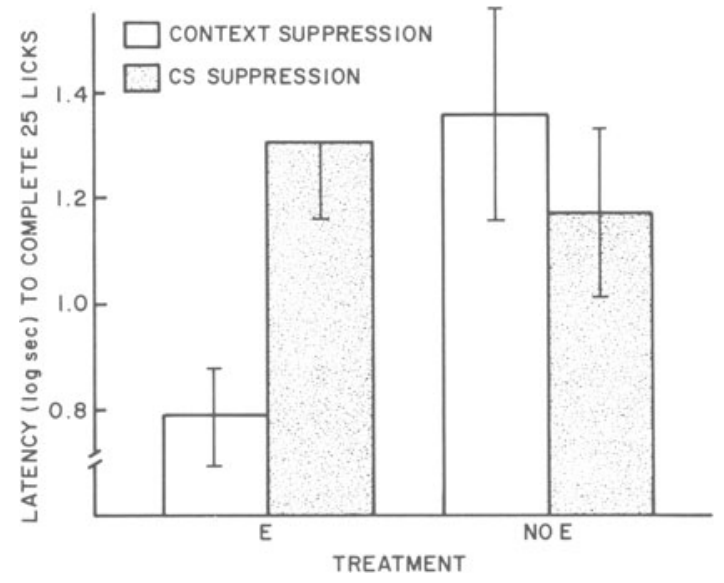

Figure 4. Mean response to the CS and to the training context in Experiment 4 after simultaneous conditioning followed by extinction (E) or no extinction (noE) of the training context. Bars indicate standard errors. 
ing. On Day 13, lick suppression in the presence of the tone CS was assessed in Context A, and on Day 14, latency to complete 25 licks in Context B was assessed.

\section{Results}

No differences in latency to complete 25 licks in Contexts A and B were noted on Day 2, or in Context A prior to CS onset on Day $13(F \mathrm{~s}<1.0)$. Groups $\mathrm{E}$ and noE did not differ in response to the tone $\operatorname{CS}[F(1,10)=0.45]$ (see Figure 4). However, Group noE did suppress more to Context $\mathrm{B}$, the training context $[F(1,10)=12.47$, $p<.01]$.

Although postconditioning extinction of the training context attenuated excitatory responding to that context, it had no effect on the response to the CS trained in that context. This suggests that, with the present parameters, the simultaneous conditioning deficit is not a performance failure mediated by the excitatory strength of the training context at the time of testing.

\section{GENERAL DISCUSSION}

In Experiment 1 we found that simultaneous presentation of a CS and US in multiple contexts enhanced responding to the CS relative to training in a single context. This was not true of a CS trained with a forward relationship to the US. Differential context-US associative strength was hypothesized to account for this difference, inasmuch as simultaneous, but not forward, pairings endowed the context with a high level of associative strength. In Experiments 2-4 we found no evidence in support of this hypothesis. In Experiment 2, we found no inverse relationship between responding to the CS and its training context as a result of varying the number of CS-US pairings. In Experiment 3, extensive preexposure to the training context attenuated the development of context-US associations during simultaneous conditioning but had no facilitatory effect on responding to the CS. Similarly, in Experiment 4, postconditioning extinction of the training context had no effect on responding to the CS. All CS testing occurred in a neutral context.

On the basis of the results of Experiments 2-4, it is not clear why enhanced responding is observed when a simultaneous CS is trained in multiple contexts. Training in multiple contexts may enhance both forward and simultaneous conditioning by reducing generalization decrement as a result of testing in a nontraining context, or by directing attention to the CS, which was common to all contexts. Enhancement of forward conditioning in Experiment 1 may have been obscured by a ceiling effect, although no animals in that study suppressed for the maximum allowable $600 \mathrm{sec}$. Other studies have shown forward conditioning to be enhanced by training in multiple contexts (Smith, 1985); it is possible that the parameters employed in Experiment 1 here simply favored this effect with a simultaneous CS, whereas other parameters may favor the effect with a forward CS. Whatever the mechanism underlying this effect, training in multiple contexts appears to be one means by which the simultaneous conditioning deficit can be reduced.

\section{REFERENCES}

Burkhardt, P. E., \& Ayres, J. J. B. (1978). CS and US duration effects in one-trial simultaneous fear conditioning as assessed by conditioned suppression of licking in rats. Animal Learning \& Behavior, 6, 225-230.

Cantor, M. B. (1981). Information theory: A solution to two big problems in the analysis of behavior. In P. Harzem \& M. D. Zeiler (Eds.), Predictability, correlation, and contiguity (pp. 287-320). New York: Wiley.

Heth, C. D. (1976). Simultaneous and backward fear conditioning as a function of number of CS-UCS pairings. Journal of Experimental Psychology: Animal Behavior Processes, 2, 117-129.

Lubow, R. E. (1973). Latent inhibition. Psychological Bulletin, 79, 398-407.

Matzel, L. D., Brown, A. M., \& Miller, R. R. (1987). Associative effects of US preexposure: Modulation of conditioned responding by an excitatory training context. Journal of Experimental Psychology: Animal Behavior Processes, 13, 65-72.

Miller, R. R., \& Schachtman, T. R. (1985). Conditioning context as an associative baseline: Implications for response generation and the nature of conditioned inhibition. In R. R. Miller \& N. E. Spear (Eds.), Information processing in animals: Conditioned inhibition (pp. 51-88). Hillsdale, NJ: Erlbaum.

Pavlov, I. P. (1927). Conditioned reflexes. Oxford: Oxford University Press.

Randich, A., \& LoLordo, V. M. (1979). Preconditioning exposure to the unconditioned stimulus affects the acquisition of a conditioned emotional response. Learning \& Motivation, 10, 245-277.

REsCorla, R. A. (1984). Signaling intertrial shocks attenuates their negative effect on conditioned suppression. Bulletin of the Psychonomic Society, 22, 225-228.

SMITH, S. M. (1985). Effects of number of study environments and learning instructions on free-recall clustering and accuracy. Bulletin of the Psychonomic Society, 23, 440-442.

(Manuscript received for publication January 4, 1988.) 